\title{
PERSPECTIVA DE NIÑOS Y NIÑAS PREESCOLARES, DE SEIS DIRECCIONES REGIONALES, ACERCA DE SUS PROCESOS EDUCATIVOS
}

\author{
Marianella Castro Pérez ${ }^{1}$ \\ Ana Cecilia González Quirós ${ }^{2}$ \\ Gina Induni Alfaro ${ }^{3}$ \\ Zulay Pereira Pérez ${ }^{4}$ \\ Willy Umaña Fernández ${ }^{5}$
}

\section{Resumen}

El presente artículo analiza la perspectiva de niños y niñas preescolares acerca de sus procesos educativos. Retoma sólo una parte de la información apor- tada mediante un Test de Oraciones Incompletas y representaciones gráficas, que forma parte de una investigación titulada Perfiles, Dinámicas y Desafios del Sistema Educativo Formal Costarricense. Dicha investigación se realizó en

1 Licenciada en Educación Preescolar, Estudios de Maestría en Administración Educativa. Experiencia como docente en educación preescolar. Docente e investigadora universitaria. Posee variadas publicaciones en el campo educativo.

2 Licenciatura en Estadística con especialización en Economía de la Universidad de Costa Rica. Estudios en el campo administrativo en el IICAP, estudios de mercadeo en el INCAE. Experiencia en docencia universitaria, en administración en el campo cooperativo y en planificación.

3 Máster en Estudios Latinoamericanos del Instituto de Estudios Latinoamericanos de la Universidad Nacional; egresada de Psicología de la Universidad de Costa Rica. Docente de la División de Educología e investigadora del Centro de Investigación y Docencia en Educación. Tiene publicaciones en los campos de la construcción de las identidades y diferencias juveniles.

4 Catedrática de la Universidad Nacional. Máster en Psicología de la Universidad de Costa Rica. Excoordinadora del Programa de Investigación en Epistemología Genética y Educación del IIMEC - UCR. Exdirectora de la División de Educación Básica de la Universidad Nacional. Docente de la División de Educación Básica e investigadora del Centro de Investigación y Docencia en Educación. Tiene publicaciones en el campo de la psicología educativa.

5 Licenciado en Administración del Trabajo con énfasis en Estudios Universitarios de la Universidad Nacional. Planificador de la Universidad Nacional; Ex Coordinador a.i. de la Oficina de Planificación de la Universidad Nacional. Miembro de diferentes comisiones institucionales e interinstitucionales; Miembro de la Comisión de Trabajos Finales de Graduación de la División de Educación para el Trabajo. Docente de la División de Educación para el Trabajo, del Instituto de Estudios del Trabajo, de la Escuela de Sociología. Tutor y lector de trabajos finales de graduación e investigador del Centro de Investigación y Docencia en Educación. Tiene publicaciones en los campos de la planificación, presupuesto. investigación, estadistica y educación. 
seis direcciones regionales educativas del país, y abarcó los niveles de preescolar, educación general básica, educación diversificada y universidad. Se trata de la primera investigación integrada entre las diferentes Unidades Académicas del Centro de Investigación y Docencia en Educación, de la Universidad Nacional y adscrita al Programa Educación y Desarrollo.

Palabras claves: preescolar, representaciones gráficas, test de oraciones incompletas.

\section{Abstract}

In this article, the authors analyze children's perspectives about their own educational processes. It introduces a brief summary of the complete research, and the data was gathered by applying an Incomplete - Sentence Test and graphic representations. This research belongs to the project "Perfiles, Dinámicas y Desafios del Sistema Educativo Formal Costarricense", and is sponsored by the CIDE (Centro de Investigación y Docencia en Educación), specifically by the program "Educación y Desarrollo". The research was developed in seven regional departments (direcciones regionales educativas), and the hole project included different educational levels: preschool, primary school, tenth and eleventh grade (educación diversificada) and university.

Keywords: children, preschool, Test and graphic representations

\section{Introducción}

T a primera investigación integrada entre las diferentes Unidades AcadéLmicas del Centro de Investigación y Docencia en Educación, de la Universidad Nacional, denominada Perfiles, Dinámicas y Desafios del Sistema Educativo Formal Costarricense, adscrita al Programa Educación y Desarrollo en Costa Rica, se realizó en seis direcciones regionales educativas del país, y abarcó los niveles de preescolar, educación general básica, educación diversificada y universitaria. Para el presente artículo se retomará únicamente una parte de la información recopilada para la educación preescolar y, más específicamente, la perspectiva de niños y niñas preescolares acerca de sus procesos pedagógicos, mediante dos instrumentos, un Test de Oraciones Incompletas y la recopilación de representaciones gráficas.

El análisis de los procesos pedagógicos desarrollados en preescolar es fundamental, de cara a evaluar, en términos de calidad y pertinencia, las acciones desarrolladas en este nivel, las que se han considerado prioridad en las políticas educativas costarricenses, desde que en 1997 se declarara obligatoria la educación preescolar. 
En el caso de preescolar, un proceso pedagógico de calidad y pertinente incluye ofrecer al niño y a la niña experiencias educativas indicadas para su desarrollo social y cognitivo, que a su vez le provean las herramientas necesarias para iniciarse en el conocimiento y actuación sobre sus espacios sociales (la familia y el jardín de infantes, por ejemplo). No menos importante, un proceso pedagógico con estas cualidades impacta positivamente en la reducción de brechas, generadas por la privación cultural y económica que sufren muchos niños y niñas.

En este sentido, indica Chaves (2002), que cuando la educación preescolar cumple a cabalidad sus funciones, las instituciones educativas para ese nivel cobran importancia en la sociedad, ayudando a la familia y estimulando el potencial de los y las infantes.

\section{Cobertura y calidad educativa de la educación preescolar}

Se ha considerado pertinente incluir una breve caracterización acerca de la cobertura y la calidad de la educación preescolar en Costa Rica, con el objetivo de que la información presentada sobre la percepción de los niños y las niñas pueda ser leída a la luz de indicadores como la tasa de escolaridad y la tasa de deserción.

En el gráfico 1, se observa que a pesar de que, constitucionalmente, el país plantea la obligatoriedad de la educación para todos, las cifras muestran una clara desigualdad entre las regiones, así como una gran cantidad de personas en condición de analfabetismo.

\section{Gráfico 1}

Analfabetismo por número de personas, según cantón visitado

(Censo 2000)

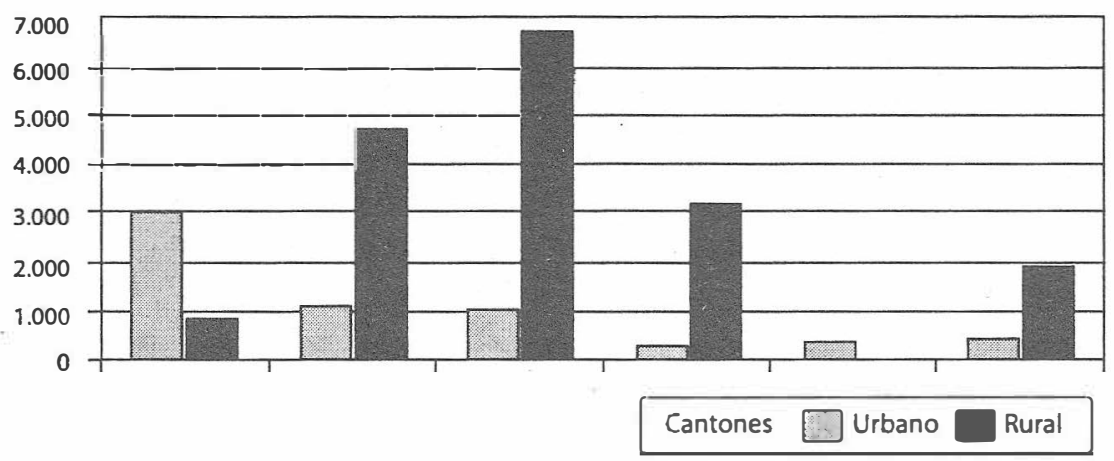

Fuente: Elaboración propia a partir de fuentes primarias. 
Las oportunidades educativas son uno de los elementos cruciales para el desarrollo de recursos y mecanismos, que permiten hacer frente a la privación cultural y económica. En este sentido, la designación temprana de programas estratégicos y de políticas educativas tendientes a fortalecer el desarrollo de los niños y las niñas con equidad y calidad, constituye una tarea política de primera importancia.

El análisis del comportamiento de la equidad, visto como acceso a la educación preescolar, revela que, en los últimos 15 años, el país ha hecho importantes esfuerzos por aumentar la cobertura educativa en este nivel. En este sentido, el XI Informe del Estado de la Nación señala que "la educación preescolar, luego de mostrar una tasa bruta de escolaridad de $62 \%$ en 1990 , alcanzó el 90.3\% en el 2004" (p. 87).

El gráfico 2 muestra información relativa al comportamiento de la tasa bruta y neta de escolaridad en el ciclo de Transición para el período 19972004. Esta serie histórica es importante, porque revela el impacto positivo que ha tenido para el país la declaración de gratuidad y obligatoriedad del ciclo de Transición, hecha en el año 1997.

\section{Gráfico 2}

Comparación tasa bruta y tasa neta de escolaridad, ciclo de transición 1997-2004

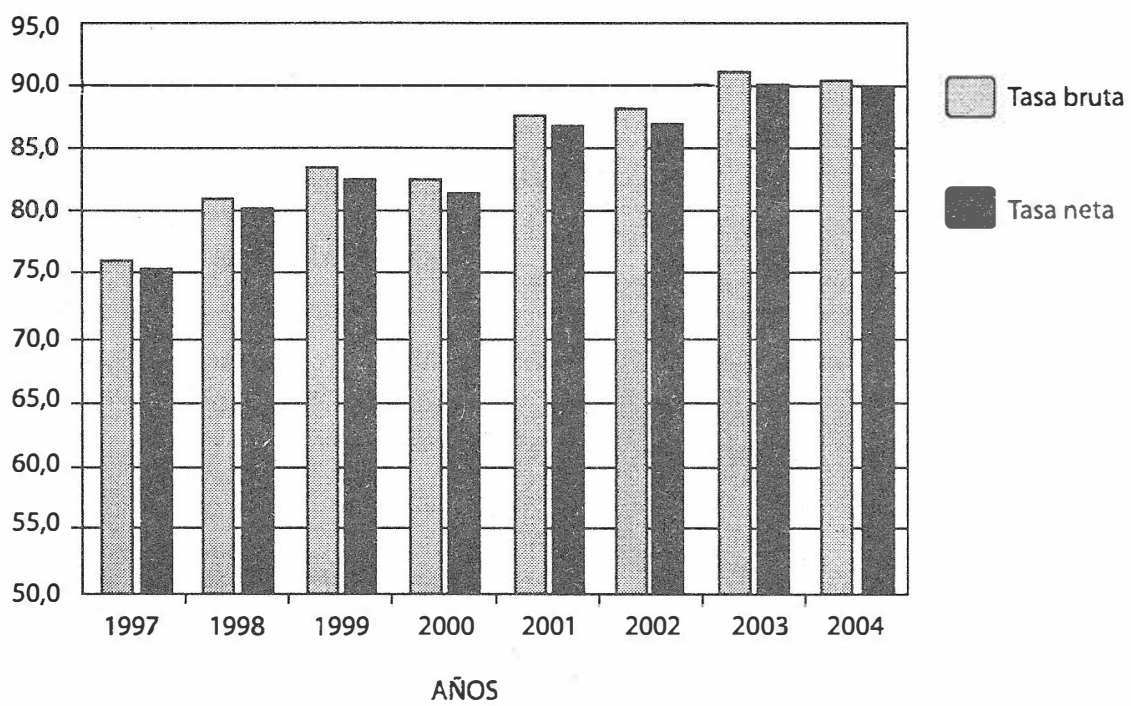

Fuente: Ministerio de Educación Pública. 
El comportamiento de la matrícula en preescolar mejora, a partir del momento en que se declara obligatorio el nivel de transición, y pasa de 63.585 estudiantes en 1997, a 72.216 estudiantes en el 2004.

La escolaridad para el ciclo de transición ha mejorado significativamente; no obstante, hay que señalar que a pesar de este avance, si se considera la normativa nacional existente, la cobertura es aún baja, ya que esta debería estar al servicio del $100 \%$ de la población.

En el cuadro 1, se presenta la matrícula en el nivel de transición en instituciones públicas y privadas en una serie histórica de 1997 al 2004.

\section{Cuadro 1}

Tasa de matrícula en Educación Preescolar, nivel de transición, de 1997 al 2004, según instituciones públicas y privadas

\begin{tabular}{|ccc|}
\hline Año & Institución pública & Institución privada \\
\hline 1997 & 56.927 & 5.885 \\
1998 & 60.694 & 5.863 \\
1999 & 62.787 & 6.145 \\
2000 & 61.710 & 6.186 \\
2001 & 66.478 & 5.748 \\
2002 & 65.956 & 5.822 \\
2003 & 67.269 & 5.730 \\
2004 & 65.539 & 5.866 \\
Tasa & $\mathbf{2 , 0 \%}$ & $\mathbf{0 , 0}$ \\
\hline
\end{tabular}

Fuente: Castro, Esquivel, González, Induni, Pereira, Solano y Umaña, 2005.

Nota: Tasa de crecimiento promedio anual 1997/2004, Modelo Geométrico.

La matrícula en preescolar en el nivel de transición, según tipo de dependencia, revela que en las instituciones privadas la matrícula ha estado estancada, pasando de 5.885 estudiantes en el año 1997 a 5.866 para el 
2004. En las instituciones públicas, la matrícula que se reporta en 1997 es de 56.927 estudiantes y de 65.539 en el 2004, para una tasa de crecimiento igual a $15,1 \%$; esto es el $2,2 \%$ en promedio anual.

Si se considera que la tasa promedio de natalidad es igual a $2,8 \%$ anual, se puede decir que es probable que muchos niños y niñas en edad de cursar la educación preescolar (ciclo de Transición), no están siendo cubiertos por el sistema educativo. Lo anterior puede ser explicado parcialmente por el hecho de que muchos padres y madres desconocen aún el carácter de obligatoriedad y gratuidad de este ciclo, o porque la oferta educativa de preescolar no se haya universalizado.

En el primer sentido, es interesante que, de acuerdo con el Censo de Población del año 2000, el 24,7\% de los padres de familia reporta que no matriculó a sus hijos en preescolar, pues sus hijos no tenían la edad requerida; no obstante, la pregunta fue hecha tomando en consideración los límites de edad estipulados por el Consejo Superior de Educación.

Finalmente, cabe señalar que aunque no hay un aumento significativo de la matrícula en el nivel de transición en instituciones privadas, estas reportan un aumento en la matrícula en niveles no formales, como maternal, interactivo I y II, ciclos en los cuales las tasas de deserción en preescolar revelan los índices más altos.

A partir de lo anterior, se puede decir que la educación preescolar enfrenta desafíos importantes relacionados con ampliar los niveles de escolaridad y promover la permanencia de niños y niñas en el sistema educativo. En cuanto al primer aspecto, las crecientes presiones económicas de los sectores medios y bajos de la población y la mayor incorporación de las mujeres en el mercado laboral, entre otras cosas, han aumentado la demanda de los padres y madres, para que sus hijos e hijas ingresen a edades más tempranas al sistema educativo.

Sin embargo, los esfuerzos se han de dirigir no sólo a ampliar las tasas de cobertura, sino también a constituirse en el objetivo de disminuir los niveles de deserción y promover, por lo tanto, la permanencia de la población infantil en el sistema educativo.

A lo largo del tiempo, la deserción en la educación preescolar ha mostrado una tasa importante (alrededor del 4\%) y viene presentando incrementos que, aunque no son significativos, sí plantean una llamada de atención. 


\section{Gráfico 3}

Evolución de la deserción en Educación Preescolar, 1998-2003

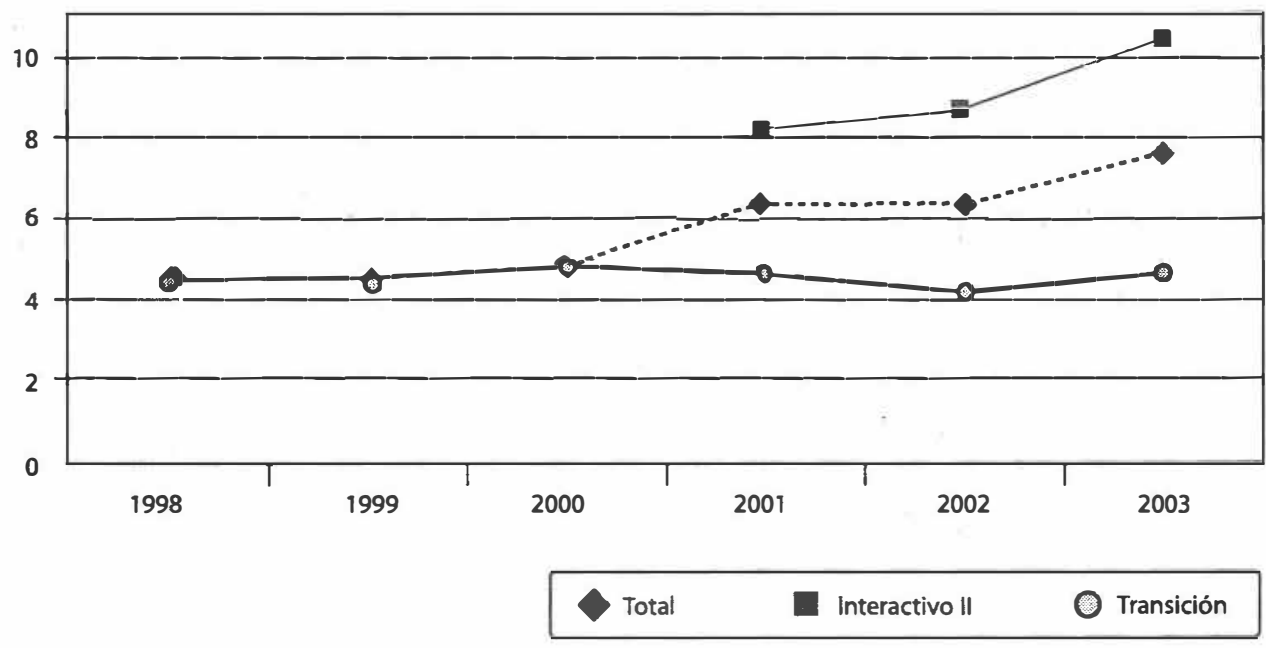

Fuente: Ministerio de Educación Pública.

Tal y como puede observarse en el gráfico 3, el comportamiento de la deserción en el ciclo de Transición, en el período comprendido entre 1998 y el 2003, muestra un incremento de 4,4\%, reportándose para $1998(4,5 \%)$, 1999 (4,5\%), 2000 (4,8\%), 2001 (4,6\%), 2002 (4,2\%) y 2003 (4,7\%). Además, la tasa de deserción es sensiblemente mayor en el Ciclo Interactivo II cuando es comparado con el de Transición.

Los datos anteriores coinciden con la evaluación realizada en el XI Informe del Estado de la Nación (2005), en el sentido de que en términos generales, en el sistema educativo costarricense coexiste con el aumento de la cobertura educativa, el incremento de la desescolarización temprana de los y las estudiantes.

Por otra parte, desde la perspectiva de esta investigación, la calidad educativa es un atributo desarrollado en todo el proceso educativo, y no en uno solo de sus componentes. Es calidad, entonces, asignar los recursos humanos, materiales e infraestructurales necesarios, desarrollar un currículo con pertinencia cultural, un proceso pedagógico que medie la construcción de aprendizajes y competencias adecuadas para habilitar a los sujetos en la comprensión y transformación de sus condiciones de vida, y por esto, con capacidad de impactar favorablemente en el logro del bienestar personal y social. 
Se destacan en el gráfico 4, algunos de los datos recopilados mediante la investigación que sirve de base a este artículo (Castro, Esquivel, González, Induni, Pereira, Solano y Umaña, 2005), y que permiten visualizar algunos aspectos referidos a la calidad de la educación preescolar, en particular al estado de los recursos en las regiones visitadas.

\section{Gráfico 4}

Estado de las aulas de Educación Preescolar, según direcciones regionales educativas (DRE) visitadas

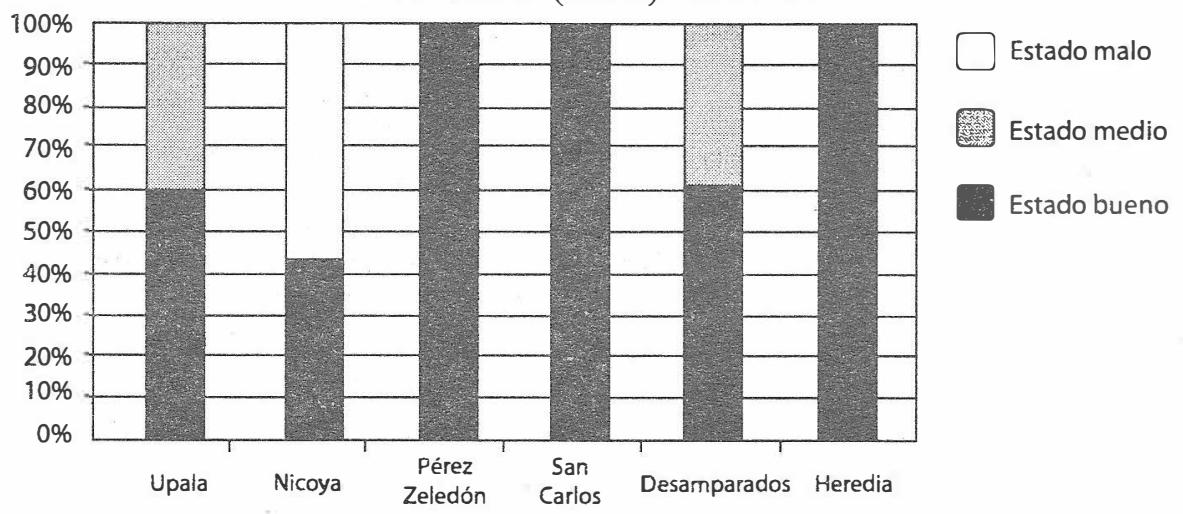

Direcciones regionales educativas

Fuente: Elaboración propia a partir de fuentes primarias.

En las instituciones visitadas, tres de seis direcciones regionales educativas se caracterizaron por disponer de aulas en "Buen estado"; estas son DRE Heredia, DRE San Carlos y DRE Pérez Zeledón. Además, dos de ellas presentaron una alta proporción de aulas de Preescolar en "Buen estado" (dos terceras partes) y la tercera parte restante en "Regular estado". Únicamente, una tercera parte de las instituciones de las DRE de Upala estaban en "Mal estado".

$\mathrm{El}$ análisis de las condiciones y servicios básicos que poseen las instituciones visitadas para el desarrollo del proceso educativo, señala que, entre otras, la modalidad de Preescolar evidencia la mayor cantidad de condiciones y servicios básicos para el quehacer educativo. Por ejemplo, el estado de las aulas, la infraestructura, la dotación de mobiliario, equipo y materiales, y la profesionalización del personal docente.

Se evidenció que las instituciones educativas donde se imparten las modalidades de Preescolar, III Ciclo y Educación Diversificada Experimental Bilingüe, mostraron las mejores condiciones en mobiliario y equipo. 
El análisis de las condiciones materiales evidenció que, las instituciones educativas donde se imparten las modalidades de Preescolar y I y II Ciclo Regular mostraron las mejores condiciones en materiales.

Adicional a lo anterior, se pudo constatar que las modalidades educativas de III Ciclo y Diversificado Científico, III Ciclo y Diversificado Técnico, Educación Especial y Preescolar y I y II Ciclos Regular, presentaron los docentes con mayor nivel académico, en comparación con las demás modalidades.

\section{Consideraciones teóricas para el estudio de las perspectivas de los niños y las niñas}

El análisis del proceso educativo es uno de los componentes más importantes para evaluar la calidad de la educación. En el caso de preescolar, lo anterior significa ofrecer al niño experiencias educativas indicadas para su desarrollo social y cognitivo.

Es interesante en este sentido, y en relación con los objetivos de la educación preescolar, el debate planteado en tomo a la escolarización temprana. Desde la perspectiva de esta investigación, no sólo es inconveniente, porque amenaza el desarrollo natural de los niños y las niñas, quienes experimentan y reconocen el mundo a través del juego, sino también porque esconde una pretensión política de grandes consecuencias. Según lo anterior, la pregunta que habría que formularse es: ¿para qué escolarizar tempranamente?

Por otra parte, investigaciones como las de Cerdas, León, Ruiz y Vidal (2005) documentan, que un número creciente de padres de familia, docentes y estudiantes de primaria y secundaria, concibe que la educación de calidad, el acceso universal a la educación (gratuita y obligatoria) y el respeto a los derechos individuales, son aspectos importantes de considerar en el campo educativo. Aspectos que denotan una vida de calidad, una formación crítica que vincule y articule la sociedad y la educación, se constituyen en elementos levemente mencionados por los informantes de la investigación. De acuerdo con esto, una educación de calidad se concibe como una educación integral, donde los valores y lo académico son esenciales.

Sin embargo, el enfoque educativo que orienta esta visión no contempla que la calidad del trabajo pedagógico implica, además, desarrollar en los niños y las niñas las herramientas necesarias para iniciarse, a tono con su desarrollo evolutivo, en el conocimiento y actuación sobre sus espacios sociales (la familia y el jardín de infantes, por ejemplo). 
Tal y como revela esta investigación, las tensiones en tomo a este debate son importantes. Al indagar con los propios niños cuál es su perspectiva del jardín de infantes, expresan que el trabajo metodológico de los docentes es tradicional y academicista, y que sus intereses, por el contrario, apuntan hacia experiencias no tradicionales y lúdicas (Castro et al., 2005).

En esta investigación, se ha optado por recurrir a las representaciones gráficas, considerando que estas constituyen un modo de expresión de los niños y las niñas, y son reflejo de sus construcciones mentales, en consecuencia, de la expresión de sus pensamientos. Los niños y las niñas preescolares no cuentan con la posibilidad de un desarrollo del lenguaje que les permita la expresión fluida de sus pensamientos; por consiguiente, en esta investigación se ha optado por recurrir a la aplicación de instrumentos, como son las representaciones gráficas y el Test de Oraciones Incompletas, medios adaptados a las características del desarrollo infantil.

Cabe destacar que tal como señalan Fontana, Pereira y Rojas (2005), el dibujo infantil no se constituye en un fin en sí mismo, sino que es un medio de expresión y de comunicación con el entorno. El dibujo le permite al niño y a la niña expresar sentimientos, emociones y conocimientos, por lo que cobra un valor incalculable como medio de expresión.

Tiraferri (s.f.) señala que por medio del dibujo

(...) el niño muestra distintos aspectos de su mundo interior, proyectando en ocasiones lo que realmente es, en otras qué le gustaria ser y al mismo tiempo, sensaciones intimas como emociones, fantasías y miedos, junto con aspectos de su personalidad y de su desarrollo evolutivo (p. 1).

La autora plantea que los infantes suelen hacer representaciones gráficas sobre la cotidianidad, expresando de esa manera sus ideales o deseos, por lo que la representación gráfica también reflejaría sus deseos o ilusiones, constituyéndose así en una expresión más de su vida, su pensamiento y su imaginación (Fontana et al., 2005).

Cristofanelli (s.f.-a) hace referencia a las diferentes funciones que presupone la expresión gráfica en los niños y las niñas, entre estas:

1. Es un acto espontáneo, que requiere cada vez mayor coordinación, beneficia la formación de la personalidad y al dominar el movimiento 
significa madurar psicomotora, intelectual y afectivamente; un ejemplo de esto se ve en las habilidades primarias de la lectura y escritura.

2. "Es un medio de comunicación interpersonal" (voluntario e involuntario, oculto, no verbal, entre otros). Funciona como descarga o "sublimación de la agresividad" (p. 1).

Por lo tanto, al observar el dibujo de un infante, se debe considerar que este ha logrado representar, de manera satisfactoria, natural y espontánea, su propia personalidad, su lenguaje consigo mismo y su percepción y relación con el mundo circundante.

Como producto del proceso de investigación realizado, se logró la recopilación de representaciones gráficas de 174 niños y niñas en edad de preescolar y los resultados de su evaluación permitieron conceptualizar las etapas del desarrollo del dibujo y los niveles de complejidad de las representaciones gráficas.

\section{Consideraciones metodológicas}

En el caso específico del trabajo con niños y niñas preescolares, se visitaron instituciones educativas tanto públicas como privadas de las siguientes direcciones regionales educativas: San Carlos, Upala, Heredia (Belén), Desamparados, Nicoya y Pérez Zeledón. Se cuenta con datos de 174 niños y niñas preescolares.

Para la recopilación de datos, se procedió de forma individual, a aplicar un Test de Oraciones Incompletas (TOI) y a la recopilación de representaciones gráficas. Cabe señalar que por tratarse de niños y niñas que aún no se expresan por escrito, la recopilación de datos se hizo de manera individual. La representación gráfica constituyó un importante recurso, desde la óptica de que el dibujo funciona como expresión básica de los pensamientos de niños y niñas.

1. El Test de Oraciones Incompletas (TOI): consiste en una serie de expresiones inconclusas que versan sobre aspectos de carácter pedagógico, las cuales se ofrecen al estudiante, quien debe completar cada frase con lo primero que piense. Se utilizó dicho instrumento, para recopilar información de los y las estudiantes de preescolar, acerca de su perspectiva sobre la educación. Se recurrió a esta prueba, pues permite plantear, de una manera simple y de fácil comprensión, aspectos referidos al campo 
educativo. Tal como en el caso de las representaciones gráficas, el TOI es una técnica que permite acceder a los informantes, con instrumentos acordes con su nivel de desarrollo y características.

En el caso de preescolar, la prueba se aplicó de manera individual. A cada niño o niña, se le formulaba oralmente la frase y la investigadora transcribía la respuesta. En total se aplicaron 174 TOI.

2. Recopilación de expresiones gráficas: esta técnica se aplicó en niños y niñas del nivel preescolar. Tuvo como propósito complementar la información recopilada con el TOI, acerca de la visualización de la institución educativa. Se les explicó en forma grupal a los y las estudiantes en qué consistía el trabajo, estableciéndose como consigna básica "dibuje cómo es el jardín de infantes". Las investigadoras supervisaron el trabajo aclarando dudas a aquellos estudiantes que no tuvieron claridad acerca de lo solicitado. En total, se recopilaron 174 representaciones gráficas.

Para el caso específico de este artículo, se retomaron las expresiones verbales y gráficas de niños y niñas preescolares, referidas a dos grandes categorías de análisis: a) motivos que generan permanencia en el sistema y b) metodología utilizada por la docente; se escogieron estas dos categorías por su valor comparativo. Los resultados pueden observarse en el siguiente análisis de los resultados.

\section{Análisis de datos}

Para el análisis de datos, se hizo una transcripción textual de las respuestas del TOI, respetándose la organización por zona, institución y ciclo. Posteriormente, se establecieron agrupaciones por elementos comunes hasta identificar categorías más específicas, que brindaron mayor precisión y que facilitaron el análisis y la comparación de los datos recopilados.

Las cinco categorías establecidas como relevantes, a partir del análisis de las respuestas de niños y niñas, fueron:

1. Opinión sobre la utilidad del jardín de infantes (el Kínder es...; mi aula es...; voy al Kínder para...). 
2. Motivos que generan permanencia en el sistema (lo que más me gusta del Kínder es...).

3. Opinión sobre el desempeño docente (mi maestra(o)...).

4. Metodología utilizada por la docente (en el aula la maestra(o) y yo hacemos...).

5. Opinión de los estudiantes acerca de la percepción familiar sobre la educación (en mi casa piensan que el Kínder...).

\section{Principales resultados del TOI}

Se presentan en la matriz 1 , los resultados obtenidos en los ítemes indicados y que permiten contrastar las opiniones de niños y niñas preescolares acerca de lo que más les gusta del jardín de infantes y lo que hacen en el aula con su maestra.

\section{Matriz 1}

Matriz comparativa de las respuestas de los niños y las niñas de preescolar sobre lo que más les gusta del jardín de infantes y lo que hacen con la maestra en el aula

\begin{tabular}{llclr} 
Categoría & $\begin{array}{l}\text { Lo que más me gusta } \\
\text { del Kínder es... }\end{array}$ & Abs. & $\begin{array}{l}\text { En el Kínder mi maestra } \\
\text { y yo hacemos... }\end{array}$ & Abs. \\
\hline Juego & $\begin{array}{l}\text { Jugar / jugar un montón / jugar } \\
\text { con mis compañeros }\end{array}$ & $\mathbf{6 0}$ & Jugar / jugamos & $\mathbf{3 7}$ \\
\hline Áreas & $\begin{array}{l}\text { Áreas / arena / arte/ casita / bi- } \\
\text { blioteca / pulpería }\end{array}$ & $\mathbf{1 8}$ & $\mathbf{0}$ \\
\hline Juguetes & Juguetes / legos / títeres & $\mathbf{1 8}$ & $\mathbf{0}$ \\
\hline $\begin{array}{l}\text { Pintar /di- } \\
\text { bujar }\end{array}$ & Pintar, dibujar / dibujamos & $\mathbf{1 7}$ & $\begin{array}{l}\text { Pintar / pintamos, dibujar / } \\
\text { dibujamos }\end{array}$ & $\mathbf{2 7}$ \\
\hline
\end{tabular}

Aprender Aprender

Aprender / para aprender cosas

13 / aprendemos / aprender cosas $\mathbf{1 8}$ nuevas / aprendemos a leer /

\begin{tabular}{llrlr}
\hline Play & Play / las hamacas / ir al play & $\mathbf{1 0}$ & $\mathbf{0}$ \\
\hline Estudiar & Estudiar & $\mathbf{8}$ & Estudiar & $\mathbf{1 0}$ \\
\hline
\end{tabular}




\begin{tabular}{llrlr} 
Categoría & $\begin{array}{l}\text { Lo que más me gusta } \\
\text { del Kínder es... }\end{array}$ & Abs. & $\begin{array}{l}\text { En el Kínder mi maestra } \\
\text { y yo hacemos... }\end{array}$ & Abs. \\
\hline Trabajos & Trabajos / trabajar & 6 & Trabajos / trabajar en el libro & 28 \\
\hline Recreo & Recreo & 6 & Recreo & 1 \\
\hline El patio & El patio / el parque & 6 & 0 \\
\hline Comida & $\begin{array}{l}\text { Comer / los helados / la comida } \\
\text { / soda }\end{array}$ & 5 & Comer & 1 \\
\hline
\end{tabular}

Maestra Estar con la teacher / la maestra $\quad 3$ La maestra me ayuda $\quad 1$

El Kínder El jardín de infantes / la escuela $\quad 3 \quad 0$

\begin{tabular}{|c|c|c|c|c|}
\hline Obedecer & Portarme bien / escuchar & 2 & $\begin{array}{l}\text { Hacer caso / estar en silencio / } \\
\text { hacer lo que la maestra dice / } \\
\text { poner atención }\end{array}$ & 7 \\
\hline Compartir & Compartir & 2 & Compartir & 3 \\
\hline Todo & & 2 & & 1 \\
\hline Rezar & Rezar & 1 & $\begin{array}{l}\text { Oraciones antes de comer / } \\
\text { rezar }\end{array}$ & 4 \\
\hline Cantar & Canciones & 1 & Cantar & 3 \\
\hline Tareas & & 0 & & 7 \\
\hline \multicolumn{2}{|l|}{$\begin{array}{l}\text { Actividades } \\
\text { matemáticas }\end{array}$} & 0 & $\begin{array}{l}\text { Sumas / contar "números en la } \\
\text { pizarra" }\end{array}$ & 6 \\
\hline \multicolumn{2}{|l|}{$\begin{array}{l}\text { Cosas muy } \\
\text { divertidas }\end{array}$} & 0 & $\begin{array}{l}\text { Cosas muy divertidas / diver- } \\
\text { timos }\end{array}$ & 3 \\
\hline \multicolumn{2}{|l|}{ Cosas } & 0 & $\begin{array}{l}\text { Cosas bonitas / importantes / } \\
\text { cosas }\end{array}$ & 3 \\
\hline \multicolumn{2}{|l|}{ Hablar } & 0 & & 3 \\
\hline
\end{tabular}




\begin{tabular}{|c|c|c|c|c|}
\hline Categoría & $\begin{array}{l}\text { Lo que más me gusta } \\
\text { del Kínder es... }\end{array}$ & Abs. & $\begin{array}{l}\text { En el Kínder mi maestra } \\
\text { y yo hacemos... }\end{array}$ & Abs. \\
\hline \multirow{18}{*}{ Otros: } & Las clases / las aulas & 2 & & 0 \\
\hline & Leer & 1 & Escribir & 1 \\
\hline & Montessori & 2 & & 0 \\
\hline & Dormir & 1 & Ponerse en el piso (reposo) & 1 \\
\hline & Las flores & 1 & Las flores & 1 \\
\hline & "Jhony me pega" & 1 & Pelear & 1 \\
\hline & Nos enseñan & 1 & Nos enseñan & 1 \\
\hline & $\therefore$ & 0 & Hace muñecos / trae cosas & 2 \\
\hline & & 0 & Ejercicio & 2 \\
\hline & Compañeros & 1 & & $\mathbf{0}$ \\
\hline & Ayudar & 1 & & 0 \\
\hline & Las fotos & 1 & & $\mathbf{0}$ \\
\hline & Bonita & 1 & & 0 \\
\hline & & 0 & Atención individual & 1 \\
\hline & & 0 & Nos dan inglés & 1 \\
\hline & & 0 & Brincar & 1 \\
\hline & & 0 & $\begin{array}{l}\text { Me siento feliz cuando estoy } \\
\text { en el jardín de infantes }\end{array}$ & 1 \\
\hline & & 0 & $\begin{array}{l}\text { Todo lo que tiene la teacher en } \\
\text { la lista }\end{array}$ & 1 \\
\hline \multicolumn{2}{|c|}{ No responde } & 0 & & 5 \\
\hline
\end{tabular}

Fuente: Elaboración propia a partir de las respuestas de niños y niñas.

\section{Lo que más me gusta del Kínder...}

Al contrastar las respuestas del TOI, ofrecidas por niños y niñas de instituciones privadas y públicas, acerca de lo que más les gusta del jardín de infantes, según la dirección regional educativa, es posible señalar que en 
términos generales, las repuestas prioritarias en las instituciones públicas son en orden descendente: el juego, los aspectos académicos y las características de la institución. Mientras que en las instituciones privadas, se señalan: las características de la institución, y en segundo lugar el juego y los aspectos académicos.

No obstante, el juego sigue siendo mencionado como un aspecto que mucho les gusta del jardín de niños, independientemente del tipo de dependencia. De los participantes, 60 respuestas están referidas al juego como lo que más les gusta.

Es interesante analizar este ítem, a la luz de las respuestas que ofrecen niños y niñas en la oración, "Voy al Kínder para...," que fue otro de los aspectos que retomó la investigación (Castro et al., 2005); mayoritariamente señalan que al jardín de niños van para realizar labores de índole académica y no lúdica. Esto indica que hay una brecha entre los deseos de los niños y las niñas propios de su desarrollo evolutivo y la vida cotidiana en las aulas de preescolar.

Existen en el país lineamientos emanados del MEP, en el sentido de no escolarizar el nivel de preescolar; no obstante, es interesante resaltar cómo en las verbalizaciones de niños y niñas, el aspecto académico, como finalidad del nivel preescolar, es el que destaca.

\section{En el aula, la maestra y yo hacemos...}

Cabe resaltar que la concreción con que se planteó a niños y niñas el ítem, pudo haberse constituido en un factor que llevara a reflejar el accionar en el aula. Es rescatable el hecho de que, tanto en dependencias públicas como privadas, salvo en pocas excepciones, se señalan preferentemente, como actividades que se realizan en el aula, las actividades tradicionales. Sin embargo, es interesante el hecho de que los niños y las niñas reportan con una alta frecuencia en sus respuestas, que en el aula con su maestra, también realizan actividades no tradicionales y lúdicas, con excepción del jardín de niños A, dependencia privada, de Desamparados, donde las verbalizaciones de estudiantes denotan una fuerte tendencia a la escolarización.

Al contrastar ambos ítemes, es posible señalar que, aunque prioritariamente los participantes señalan que lo que más les gusta del jardín de niños es jugar (60 respuestas), sólo 37 respuestas hacen alusión a que con la maestra juegan. 
En orden descendente, se observa que en segundo lugar, niños y niñas reportan que las áreas y los juguetes es lo que más les gusta del jardín de infantes; al contrastar estas respuestas, con lo que en segundo lugar se menciona que hacen en el aula con la maestra, aparecen "trabajos", "trabajar en el libro", "aprendemos a leer", actividades todas que hacen pensar más en procesos escolarizantes que lúdicos. Lo que más se acerca a procesos lúdicos es la mención que hacen a "dibujar" en el aula con la maestra, lo que también es mencionado en tercer lugar como lo que más les gusta del jardín de infantes.

\section{Temáticas de las representaciones gráficas}

La matriz 2 muestra un panorama de las representaciones gráficas realizadas por niños y niñas de las seis direcciones regionales educativas. Para efectos de análisis, se reagruparon todas las representaciones independientemente del tipo de institución (privada o pública). El análisis se hizo en función de las temáticas que presentaban los dibujos de niños y niñas.

\section{Matriz 2}

Matriz comparativa de las representaciones gráficas de niños y niñas participantes, según temática y dirección regional educativa

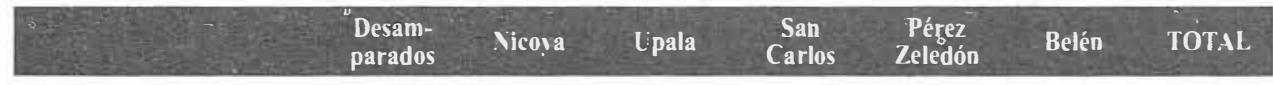

Estructura física

$\begin{array}{lrrrrrrr}\text { Del jardín de niños } & 21 & 20 & 15 & 18 & 21 & 5 & 100 \\ \text { Del aula } & 2 & 1 & 4 & 6 & 8 & 3 & \mathbf{2 4}\end{array}$

Espacios recreativos

\begin{tabular}{lrrrrrrr} 
Internos al aula & 18 & 9 & 1 & 11 & 9 & 1 & 49 \\
Externos al aula & 8 & 1 & 3 & - & 1 & 2 & $\mathbf{1 5}$ \\
\hline Familia & 3 & 17 & 7 & 8 & - & 3 & $\mathbf{3 8}$ \\
\hline Pares & 6 & 7 & 4 & 3 & 4 & -- & $\mathbf{2 4}$ \\
\hline Comunidad & 1 & -- & 1 & 1 & 3 & -- & $\mathbf{6}$ \\
\hline
\end{tabular}




\begin{tabular}{|c|c|c|c|c|c|c|c|}
\hline $0 \quad 3$ & $\begin{array}{l}\text { Desam- } \\
\text { parados }\end{array}$ & Vicoia & Lpala & $\begin{array}{l}\text { San } \\
\text { Carlos }\end{array}$ & $\begin{array}{l}\text { Pérez } \\
\text { Zcledón }\end{array}$ & Bclén & TOTAL \\
\hline Docente & 4 & 3 & 1 & 2 & 5 & 1 & 16 \\
\hline Letras y números & 6 & 7 & 3 & 1 & 6 & 1 & 24 \\
\hline $\begin{array}{l}\text { Mezcla de } \\
\text { expresiones }\end{array}$ & 6 & 23 & 12 & 12 & 12 & 5 & 70 \\
\hline $\begin{array}{l}\text { Incorporación } \\
\text { propia en el } \\
\text { dibujo }\end{array}$ & 12 & 7 & 1 & 3 & 4 & - & 27 \\
\hline
\end{tabular}

Fuente: Elaboración propia a partir de las respuestas de niños y niñas.

De manera general, es posible mencionar que las representaciones gráficas analizadas, muestran en cuanto a las temáticas, un predominio de la estructura física del jardín de infantes, tanto en hombres (52) como en mujeres (43) y la mezcla de expresiones gráficas o de elementos incorporados en el diseño que no se vinculan directamente con la estructura del jardín de infantes (37 son respuestas dadas por hombres y 33 por mujeres). En dicha categoría, fueron reagrupadas expresiones gráficas tales como: "animales", “elementos de la naturaleza", "bandera de Costa Rica”, "juguetes”, entre otros.

En los hombres, se destaca la frecuencia (31) con que dibujan los espacios recreativos externos al aula, no así en las mujeres (17).

La temática de hogar y familia parece ser muy importante para niños y niñas, más allá de sus pares o su propia incorporación en la representación gráfica solicitada. Es así como 20 mujeres destacan la casa y 4 la estructura de su grupo familiar. Mientras que en el caso de los hombres 7 dibujan la casa y 6 la estructura del grupo familiar.

Con muy baja frecuencia, sus representaciones gráficas hacen referencia al docente $u$ otros funcionarios del centro educativo, ya que sólo 8 hombres y 8 mujeres, dibujan al "docente", "director" o "guarda".

Cabe destacar también que hay dentro de las temáticas, la incorporación de letras o números en sus representaciones, existen 9 hechas por varones y 15 por mujeres.

Llama la atención al realizar el análisis de las representaciones, el hecho de que tanto niños como niñas, dibujan más los espacios externos al aula que los internos, es decir, dibujan más la estructura física del jardín de infantes (en general 95 preescolares) y los espacios recreativos externos al aula que representan 48 preescolares. La estructura física del aula es representada 
por 21 preescolares y sus espacios internos, 12 preescolares. Es posible señalar, que hay una mayor atención de niños y niñas depositada en los aspectos externos al aula, a pesar de que es en esta donde transcurre la mayor parte de su tiempo.

La referencia a la comunidad es realmente escasa ( 4 niños, 2 niñas), en dicha categoría se contemplaron "calles" y "carros".

Lo mismo sucede con la referencia a los "pares", la cual sólo es representada por 14 niños y 10 niñas, a pesar de que podría ser esperable que el interés por sus iguales estuviera representado.

\section{Consideraciones finales}

De manera muy general, puede señalarse, que los datos aportados por la investigación realizada indican que la educación preescolar ha sido una prioridad últimamente, y que en concordancia con ello, se han asignado recursos a las instituciones; esto permite señalar que, en general, los servicios de educación preescolar, de las direcciones regionales educativas visitadas, cuentan con la mayor cantidad de condiciones y servicios básicos para el quehacer educativo; tales lugares mostraron las mejores condiciones en mobiliario y equipo, así como en materiales; muy diferente a lo encontrado en otros niveles educativos.

El análisis de las dos categorías que se han retomado en el presente artículo indica que, mayoritariamente, las perspectivas que crean los niños y niñas acerca de lo que más les gusta del jardín de infantes no se cumplen cabalmente en la práctica. Ellos y ellas valoran al jardín de niños en la medida en que se constituye en un lugar donde se puede jugar, ir a áreas, usar juguetes, entre otras respuestas. Sin embargo, dichas expresiones no son coincidentes con lo planteado como acciones o actividades que realizan en conjunto con la docente, en las dinámicas de aula. De manera prioritaria, se destacan aspectos más academicistas, tendientes al uso del texto, aprender, pintar, dibujar, entre otros aspectos.

Al contrastar las respuestas dadas en el TOI y las temáticas de las representaciones gráficas, es posible indicar que como patrones, se dan los siguientes:

1. Tendencia a resaltar aspectos referidos a la estructura física de la institución educativa y del aula.

2. Prioridad concedida a los espacios recreativos externos por su valor lúdico. 
3. Tendencia a destacar lo lúdico en cuanto a espacios recreativos, juegos, áreas, juguetes.

4. Destacar actividades de carácter académico como: sumar, leer, escribir, hacer números, seguir el libro, hacer letras, entre otras.

5. Está invisibilizada la función docente y no parece ser un referente importante para niños y niñas; el docente es poco mencionado y dibujado.

6. En ambos casos (TOI y representaciones gráficas), los pares son poco mencionados y dibujados.

Es importante, por lo tanto, profundizar, en futuras investigaciones, para identificar de manera más precisa las acciones y estrategias que se desarrollan en las aulas preescolares y que bien podrían no estar siendo compatibles con las habilidades y necesidades propias del desarrollo de niños y niñas preescolares.

Por otra parte, considerando que las oportunidades educativas son uno de los elementos cruciales para el desarrollo de recursos y mecanismos por los cuales se pueda hacer frente a la privación cultural y económica, es importante reforzar la designación temprana de programas estratégicos y de políticas educativas tendientes a fortalecer el desarrollo de los niños y las niñas con equidad y calidad. 


\section{Referencias}

Castro, M.; Esquivel, L.; González, A.; Induni, G.; Pereira, Z.; Solano, J. y Umaña, W. (2005). Perfiles, Dinámicas y Desafios del Sistema Educativo Formal Costarricense. Informe Final de Investigación no publicado. Universidad Nacional, Heredia, Costa Rica.

Cerdas, V.; León, A. T.; Ruiz, S. y Vidal, M. (2005). Elementos a tomar en cuenta en un proceso de reforma educativa, desde la perspectiva de los/as estudiantes, los/as docentes y los padres y madres de familia. Informe Final de Investigación no publicado. Universidad Nacional, Heredia, Costa Rica.

Cristofanelli, P. (s.f.a). Mundo Garabato: los niños y la grafología (1). La postura de los adultos frente al garabato y el dibujo infantiles. Disponible en: http:// www.naturino.com/esp/grafologial_es.htm

Cristofanelli, P. (s.f.b). Mundo Garabato: los niños y la grafología (2). Garabato / Dibujo espontáneo: instrumento de formación y medio de comunicación. Disponible en: http://www.naturino.com/esp/grafologia2_es.htm

Fontana, A.; Pereira, Z. y Rojas, D. (2005). El concepto de esquema corporal desde la perspectiva de niños de 0 a 10 años, con y sin necesidades educativas especiales. Informe Final de Investigación no publicado. Universidad Nacional, Heredia, Costa Rica.

Instituto Nacional de Estadística y Censos (INEC). (2000). LX Censo Nacional de Población. San José, Costa Rica: Instituto Nacional de Estadística y Censos.

Ministerio de Educación Pública (MEP). (2000). Memoria institucional Año 2000. San José, Costa Rica: OFICINA DE PUBLICACIONES.

Programa Estado de la Nación. (2005). XI Informe Estado de la Nación en Desarrollo Humano Sostenible. San José, Costa Rica: PROGRAMA ESTADO DE LA NACIÓN.

Tiraferri, L. (s.f.). Dime qué dibujas... y sabré qué sientes. Disponible en: http:// www.educacion.1243.cl/papas/cero_drama/que_diujas.htm 